\title{
Use of High Sensitivity Bolometers for Astronomy: Planck High Frequency Instrument
}

\author{
J. M. Lamarre ${ }^{1}$, M. Piat ${ }^{1}$, P. A. R. Ade ${ }^{2}$, J. Bock $^{3}$, P. De Bernardis ${ }^{4}$,

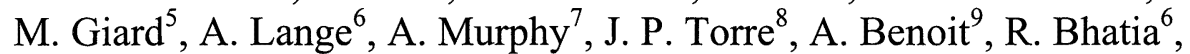 \\ F. R. Bouchet ${ }^{10}$, B. Maffei ${ }^{2}$, J. L. Puget ${ }^{1}$, R. Sudiwala ${ }^{2}$, V. Yourchenko ${ }^{7}$ \\ IInstitutd'Astrophysique Spatiale,Orsay,France,email:lamarre@ias.fr,puget@ias.fr,piat@ias.fr \\ ${ }^{2}$ CardiffUniversity,Wales, UK, email:P.A.R.Ade@qmw.ac.uk,bruno.maffei@qmw.ac.uk \\ ${ }^{3}$ Jet Propulsion Laboratory, Pasadena, Ca, USA, email: james.Bock@jpl.nasa.gov \\ ${ }^{4}$ Universita La Sapienza, Roma, Italy, email: debernardis@romal.infn.it \\ ${ }^{5}$ CESR, Toulouse, France, email: martin.giard@cesr.fr \\ ${ }^{6}$ Caltech, Pasadena, Ca,USA, email: ael@astro.caltech.edu, rsb@astro.caltech.edu \\ ${ }^{7}$ University of Maynooth, Ireland, email: amurphy@may.ie,vyourchenko@may.ie, \\ ${ }^{8}$ Service d'Aéronomie, Verrières le Buisson, France, email: Jean-Pierre.Torre@aerov.jussieu.fr \\ ${ }^{9}$ CRTBT, Grenoble, France, email: benoit@labs.polycnrs-gre.fr, \\ ${ }^{10}$ Institut d'Astrophysique de Paris, France, email: bouchet@iap.fr
}

\begin{abstract}
The Planck satellite is dedicated to the measurement of the anisotropy of the Cosmic Microwave Background (CMB) with unprecedented sensitivity and angular resolution. It is a project of the European Space Agency based on a wide international collaboration, including United States and Canadian laboratories. The detectors of its High Frequency Instrument (HFI) are bolometers cooled down to $100 \mathrm{mK}$. Their sensitivity will be limited by the photon noise of the CMB itself at low frequencies, and of the instrument background at high frequencies. The requirements on the measurement chain are directly related to the strategy of observation used for the satellite. This impacts the bolometer design as well as other elements: The cooling system must present outstanding temperature stability, and the amplification chain must show a flat noise spectrum down to very low frequencies.
\end{abstract}

\section{INTRODUCTION}

The Cosmic Microwave Background (CMB) is the most distant, and therefore the most ancient source of radiation that can be directly observed from Earth in any frequency range. The satellite COBE has measured its submillimeter emission, which is that of a nearly perfect blackbody at $2.73 \mathrm{~K}$. This emission is attributed to the primordial universe when it was about 300000 years old and warm enough $(3000 \mathrm{~K})$ to ionize the hydrogen gas that constitutes most of its mass. Due to the expansion of the universe, this radiation was red-shifted by Doppler effect by a factor of about 1000 , and thanks to the cooling due to the expansion, it could travel and reach us through the very transparent neutral hydrogen. The discovery of the CMB and its refined observation by COBE are pillars that support the big bang theory. The CMB is isotropic over the sky down to a level of $10^{-5}$ at small scales. The tiny deviations from

CP605, Low Temperature Detectors, edited by F. S. Porter et al. (C) 2002 American Institute of Physics 0-7354-0049-0/02/\$19.00 

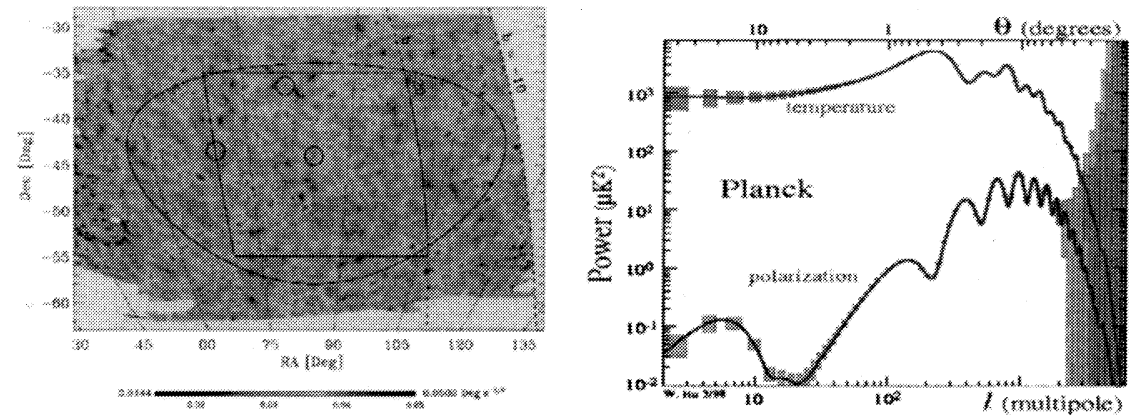

FIGURE 1. Left (a): Map of the Cosmic Microwave Background obtained by the BOOMERanG experiment. The one degree like bumps of the CMB emission are tiny local fluctuations of the brightness temperature of the CMB. Right (b): Simulation of the Planck capability in the domain of angular frequencies. Smaller details will be measured with a much better sensitivity (with respect to BOOMERanG) on the whole sky. The position of the first peak informs us on the curvature of the universe, while the whole spectrum give much more about other cosmological parameters.

uniformity give us unique information on the physics of the primeval universe, on the cosmological parameters describing the geometry of the universe, and on the history of matter and radiation since the Big Bang.

New results from the balloon-borne experiments BOOMERanG [1] and MAXIMA [2] and from ground-based experiments using radio detectors and interferometry $[3,4]$ were recently published. They gave a first view of the small scale anisotropy of the $\mathrm{CMB}$, unveiling the predicted peaks in the power spectrum of its angular distribution. The BOOMERanG CMB map shown in figure 1(a) is a real picture of our primeval universe when it became transparent to infrared radiation, and figure 1(b) shows its predicted spectral distribution in spherical harmonics, with the errors bars expected from the Planck-HFI Project.

The project Planck of the European Space Agency is intended to be, after COBE and MAP [5], the next generation of CMB experiments, pushing to its limits the knowledge that will be retrieved from the $\mathrm{CMB}$ observation with unprecedented angular resolution and sensitivity.

\section{HFI SCIENTIFIC CAPABILITIES}

The six spectral bands of the High Frequency Instrument (HFI, see Table 1) cover the frequency range between 100 and $1000 \mathrm{GHz}$ with an angular resolution of about 5 arcmin. Its sensitivity will be limited, in the CMB channels, by the statistical fluctuations [6] of the CMB itself [7], which makes it a kind of ultimate experiment. It will also measure the polarization of the $\mathrm{CMB}$ in three channels, which will give independent and unique information on the CMB anisotropy [8].

This kind of accuracy on the CMB can be achieved only by removing the various foregrounds formed by the evolving universe situated between us and the warm primordial universe emitting the CMB. Among these, we see the emission of dust and gas in our own galaxy and from other galaxies. Clusters of galaxies, that contain high 
TABLE 1. Planck HFI performances.

\begin{tabular}{|c|c|c|c|c|c|c|c|}
\hline Central Frequency & $(\mathbf{G H z})$ & 100 & 143 & 217 & 353 & 545 & 857 \\
\hline Beam Full width Half Maximum & $\operatorname{arcmin}$ & 9.2 & 7.1 & 5.0 & 5.0 & 5.0 & 5.0 \\
\hline Number of unpolarized detectors & & 4 & 4 & 4 & 4 & 4 & 4 \\
\hline Number of polarized detectors & & - & 8 & 8 & 8 & - & - \\
\hline Total sensitivity $(\partial \mathrm{T} / \mathrm{T})$ & $\mu \mathrm{K} / \mathrm{K}$ & 2.2 & 2.4 & 3.8 & 15 & 17 & 8000 \\
\hline $\mathrm{U}$ and $\mathrm{Q}$ sensitivity $(\partial \mathrm{T} / \mathrm{T})$ & $\mu \mathrm{K} / \mathrm{K}$ & - & 4.8 & 7.6 & 30 & - & - \\
\hline Flux sensitivity & mJy & 9.0 & 12.6 & 9.4 & 20 & 46 & 52 \\
\hline
\end{tabular}

temperature gas detected in the X-rays, distort the $\mathrm{CMB}$ by inverse Compton scattering. This is the Sunyaev-Zeldovich Effect (SZE), that makes clusters of galaxies good tracers of the dynamics of the universe at large scales.

Six bands in the HFI and four more in the LFI are needed to separate these various components thanks to their spectral and spatial signature. An additional benefit of the increase in complexity resulting from this approach is that all these astrophysical sources will be known much better, which is in many cases of major interest for astronomy. Planck has to be considered not only as the third generation of CMB satellites, but also as the first sub-Terahertz sky survey of modern astronomy. Several thousands of galaxies, of young stellar objects, of clusters of galaxies will be observed in a new way. Nearly every field of astronomy will benefit from its results, from the study of the solar system (Trans-Neptunian objects for example) to the large scale structure of the universe (SZE results), as well as new insight on the cold components of galaxies, a possible candidate for dark matter. The Planck project is committed to deliver a set of well-defined products to the scientific community at large.

\section{REQUIREMENTS AND DESIGN OF HFI DETECTION SYSTEM}

Planck will be put in a "halo" orbit around the L2 Lagrangian point of the SunEarth system, at about 1.5 million $\mathrm{km}$ of the Earth (Figure 2). The satellite will rotate at 1 RPM around an axis nearly anti-solar, allowing its field of view to scan large

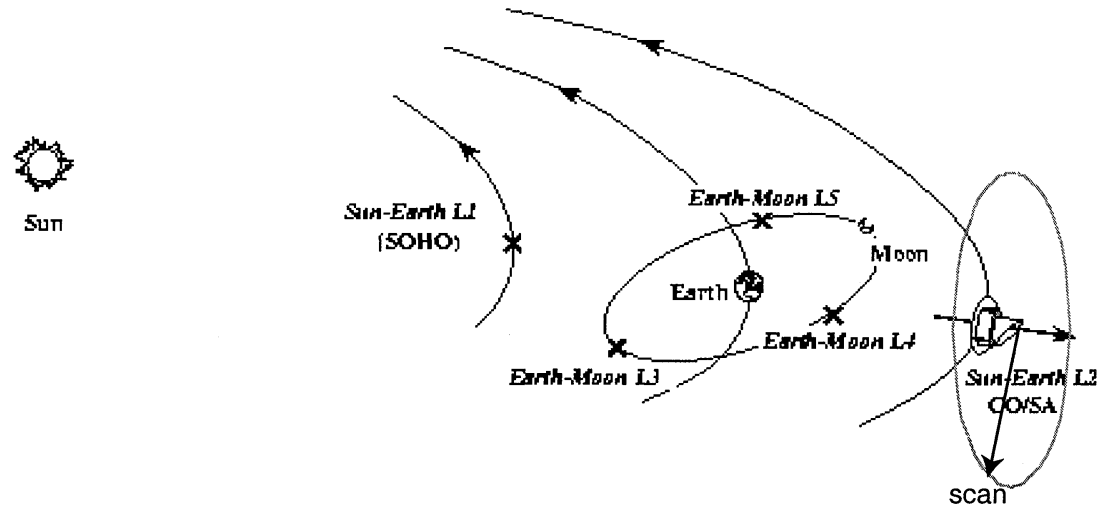

FIGURE 2. Schematic representation of the Planck orbit and scanning strategy. 


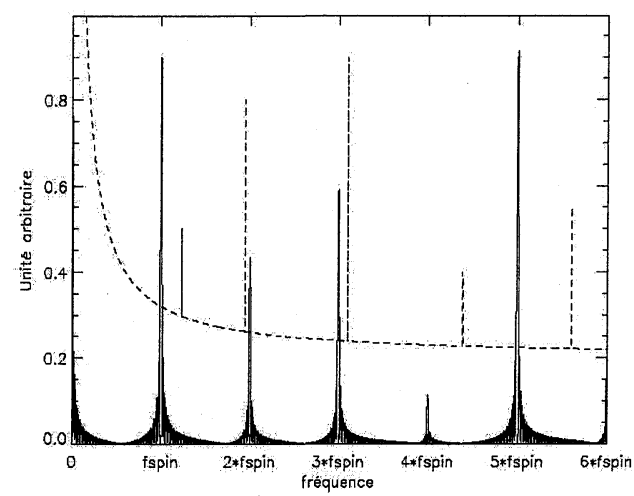

FIGURE 3. The scientific signal is periodic at the frequency of the spin rate of the satellite (1 RPM). The first 5000 harmonics contain useful information, which determines the working frequency range of the bolometers: from $0.016 \mathrm{~Hz}$ to about $100 \mathrm{~Hz}$.

circles in the sky. Every $60 \mathrm{~min}$, the axis of rotation will be shifted by 2.5 arcmin, in order to follow the movement of the Earth around the Sun. In six months the successive circles will cover the full sky.

The useful data from the telescope is therefore a quasi-periodic signal, with a period of one minute. The signal from the detection chain must therefore be stable from one observation of a source to the next one [9]. Lower frequencies can be filtered out in the data reduction process. This stability is a major requirement for the cryogenics and the electronics of HFI. The scan rate is $6 \mathrm{deg} / \mathrm{s}$. With $5 \mathrm{arcmin}$ beams, the response time of the bolometers must be less than a few milliseconds (see Table 2). This is the driving requirement for the choice of the bolometers. In particular, it was understood from the beginning of the project that only very low temperature (100 $\mathrm{mK}$ or less) would allow to reach this speed for the large bolometers needed in the millimeter range. This choice is consistent with the requirement that the sensitivity must be limited by the photon noise in the HFI wavelength range.

The absorber of the bolometers is of the spider web type [10] for unpolarized detectors. For polarized channels, the bolometers are sensitive to polarization thanks to an absorber made with parallel wires (J. Bock). NTD Ge thermometers with

TABLE 2. Required bolometer performances.

\begin{tabular}{ccccc}
\hline $\begin{array}{c}\text { Frequ. } \\
(\mathbf{G H z})\end{array}$ & $\begin{array}{c}\text { Optical Load } \\
(\mathbf{p W})\end{array}$ & $\begin{array}{c}\text { Maximum NEP } \\
\mathbf{1 0}^{-17} \mathbf{W H z}\end{array}$ & $\begin{array}{c}\text { Goal time } \\
\text { constant (ms) }\end{array}$ & $\begin{array}{c}\text { Maximum time } \\
\text { constant (ms) }\end{array}$ \\
\hline $\mathbf{1 0 0}$ & 1.0 & 1.2 & 3.9 & 7.8 \\
$\mathbf{1 4 3}$ & 1.1 & 1.5 & 2.9 & 5.7 \\
$\mathbf{2 1 7}$ & 1.1 & 1.8 & 2.2 & 4.4 \\
$\mathbf{3 5 3}$ & 1.0 & 2.2 & 2.2 & 4.4 \\
$\mathbf{5 4 5}$ & 5.0 & 6.0 & 2.2 & 4.4 \\
$\mathbf{8 5 7}$ & 16.0 & 13.5 & 2.2 & 4.4 \\
$\mathbf{1 4 3 P}$ & 0.57 & 1.1 & 3.0 & 5.7 \\
$\mathbf{2 1 7 P}$ & 0.54 & 1.3 & 2.2 & 4.4
\end{tabular}



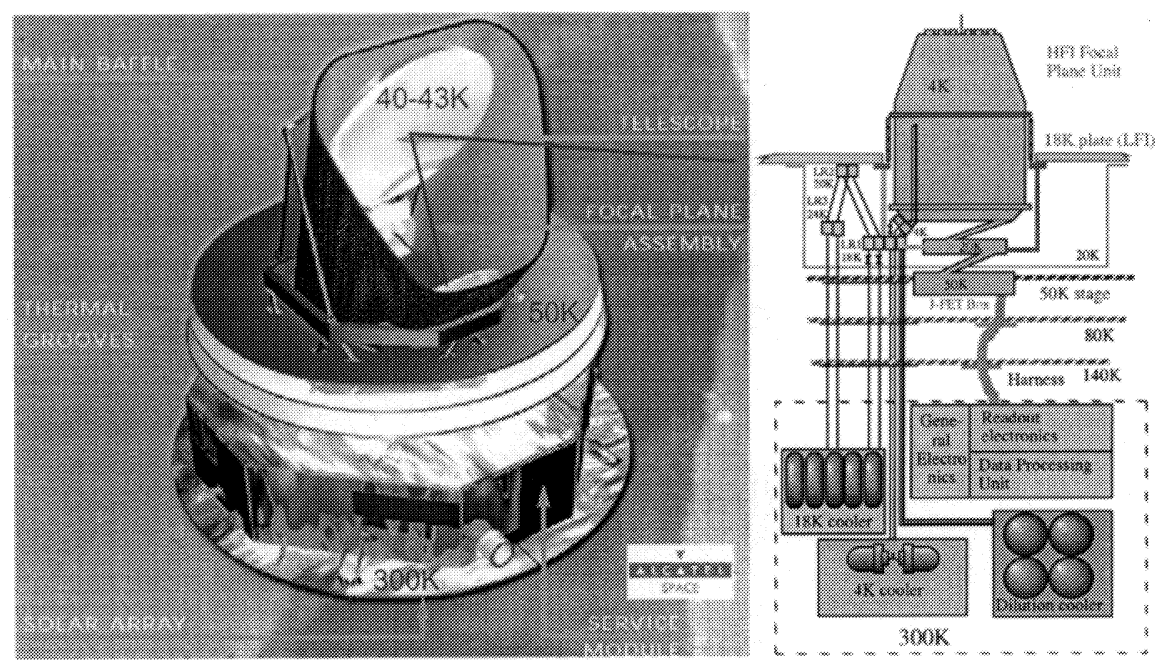

FIGURE 4. Left: The solar panels cover the bottom part of the satellite. The thermal architecture allows to passively cool the telescope down to $40 \mathrm{~K}$. Right: Three differrent active coolers are needed to cool the bolometers down to $0.1 \mathrm{~K}$.

impedance of about $10 \mathrm{M} \Omega$ are the sensitive elements. The required performances are listed in Table 2.

The readout electronics must not be the limiting factor of the detection chain. A specially developed amplifier [11] using tunable AC bias and cold J-FET preamplifier shows a $5 \mathrm{nVHz}^{-1 / 2}$ noise down to $10^{-2} \mathrm{~Hz}$.

The Planck design is driven by the thermal requirements. Thanks to its stable orientation with respect to the sun, the moon and the earth, it was possible to obtain an efficient passive cooling of the science payload down to $50 \mathrm{~K}$ or less (see Figure 4).

J-T expansion of hydrogen using sorption pumps cools both LFI and HFI down to $20 \mathrm{~K}$. Mechanical compressors provide helium for the $4 \mathrm{~K} \mathrm{~J}$-T compressor. The open loop helium $3 /$ helium 4 dilution cooler provides both the cooling of the bolometers down to $100 \mathrm{mK}$ and cools an intermediate stage at $1.6 \mathrm{~K}$ by J-T expansion of the helium mixture. The internal architecture of the HFI focal plane unit is shown on Figure 5. As shown on the right section of this figure, radiation from the various stages loads the bolometers. This radiation must be very stable, because any fluctuation in the range of useful frequencies $(0.016$ to $100 \mathrm{~Hz})$ could be taken as coming from the sky. This implies very good temperature stability of the various stages of the HFI-focal plane unit: $20 \mathrm{nkHz}^{-1 / 2}$ for the $100 \mathrm{mK}$ stage (directly connected to the bolometers), $28 \mu \mathrm{KHz}^{-1 / 2}$ for the $1.6 \mathrm{~K}$ stage, and $10 \mu \mathrm{KHz}^{-1 / 2}$ for the $4 \mathrm{~K}$ stage.

\section{CONCLUSIONS}

Fundamental fluctuations of the flux reaching the detectors are the main parameter limiting the instrument sensitivity. Photon noise of the CMB radiation itself limits the 

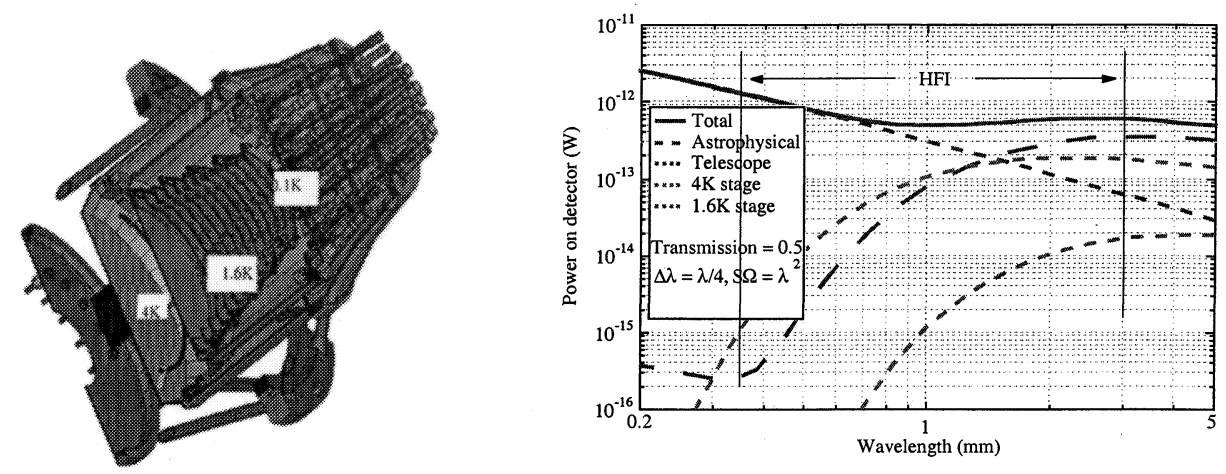

FIGURE 5. The focal plane unit of the HFI consists of three stages at $4 \mathrm{~K}, 1.6 \mathrm{~K}$, and $0.1 \mathrm{~K}$. The coupling of the bolometer with the telescope is made thanks to corrugated horns at $4 \mathrm{~K}$. These stages radiate on the detectors and contribute to the background as well as the telescope (right).

sensitivity in the three most sensitive channels. The scanning strategy plays also a major part in the derivation of the bolometer requirements, since it gives, for signal and noises, the relation between the time domain and the domain of angular scales. A study of the bolometer requirements in the Fourier domain proved to be an efficient tool for several aspects of the instrument design.

Planck HFI is now under construction, for a launch in early 2007. We thank all the technical and scientific persons who contributed to the design of this instrument.

\section{REFERENCES}

1. P. de Bernardis et al., A flat universe from high resolution maps of the CMBR, Nature, v404, 2000, p. 955

2. A. T. Lee et al., A high spatial resolution analysis of the MAXIMA-1 cosmic microwave background anisotropy data, astro-ph/0104459

3. A. D. Miller et al, A Measurement of the Angular Power Spectrum of the CMB from $l=100$ to 400, Accepted by ApJL, Astro-ph/9906421

4. N. W. Haverson et al., DASI first results: A Measurement of the Cosmic Microwave background Angular Power Spectrum, Astro-ph/0104489

5. M. Halpern and D. Scott, Future Microwave Background Experiments, Astro-ph/9904188

6. J. M. Lamarre, Photon Noise in Photometric Instruments at Far Infrared and Submillimeter Wavelengths, Appl. Opt. 25, 870 (1986)

7. J. M. Lamarre et al., The High Frequency Instrument of PLANCK: Design and Performances, Astro. Lett. And Communications, vol. 37, pp.161-170 (2000)

8. F. R. Bouchet, J. L. Puget, J. M. Lamarre, The cosmic microwave background: from detector signals to constraints on the early universe physics, in The primordial universe, Binettruy et al. Editors, EDP sciences, Les Ulis, Paris, 2000, pp. 103-220

9. M. Piat et al., Proceedings of LTD8, Dalfsen, the Netherlands, 1999, NIMA, 444, pp. 419-422 (2000).

10. A. D. Turner et al., $\mathrm{Si}_{3} \mathrm{~N}_{4}$ micromesh bolometer array for sub-millimeter astrophysics, accepted for publication in Applied Optics, 2001.

11. S. Gaertner et al., A new readout system for bolometers with improved low frequency stability, Astron. \&Astrophys. Sup, 126, 151-160, 1997. 